\title{
La Dame de Lespugue et les avatars d'une femme de la préhistoire revisitée
}

\author{
The Lady of Lespugue, or Avatars of a prehistoric woman revisited
}

\author{
Patrick Paillet $^{1}$ \\ ${ }^{1}$ Préhistorien et Maître de conférences HDR du Muséum national d'Histoire naturelle, Département Homme \& \\ Environnement, UMR Histoire naturelle de l'Homme préhistorique 7194, équipe Nomade, Musée de l'Homme.
}

RÉSUMÉ. La «Vénus » ou plutôt, devrait-on dire, la « Dame » de Lespugue exhibe un corps dénudé dont les formes hypertrophiées, nées dans l'ivoire par le truchement de l'esprit et de la main d'un sculpteur de génie, ont nourri depuis sa découverte une abondante littérature scientifique, artistique et même poétique. Si la statuaire préhistorique nous réserve parfois des surprises esthétiques, soit parce ce qu'elle affiche ses prétentions naturalistes, soit parce qu'elle revendique une expression délibérément schématique, offre peu souvent une telle modernité expressive. On a tant dit et tant écrit sur cette vieille dame, dont les bonnes manières voudraient que nous taisions l'âge, mais qui affiche tout de même environ 25000 ans, que le souffle d'éternité qui l'anime viendrait à disperser notre inspiration. Mais on ne saurait rester coi face à cette représentation idéelle de la femme qui défie le bon sens anatomique. Nous proposons dans ces quelques lignes de lever un coin du "voile " ou plutôt, devrait-on dire, du " pagne » qui n'a jamais rien caché de sa nudité réinventée.

ABSTRACT. The "Venus", or as we should say, the "Lady" of Lespugue displays a naked body whose hypertrophied forms, born from ivory through the mind and hand of a sculptor, have since its discovery contributed to an abundant scientific, artistic and even poetic literature. If prehistoric statuary sometimes has some aesthetic surprises for us, either because it displays its naturalistic pretensions, or because it claims a deliberately schematic expression, it seldom offers such an expressive modernity. So much has been said and written about this old lady - good manners should keep us silent about her age - but even after about 25,000 years, she still shows that the breath of eternity which animates her could fire up our inspiration. But we cannot remain coy in the face of this ideal representation of a woman that defies all anatomical sense. Therefore, we propose in these few lines to lift a corner of the "veil" or the "loincloth" which has never hidden anything of its reinvented nudity.

MOTS-CLÉS. Art, Préhistoire, Vénus, Femme, Lespugue, Ronde-bosse, Ivoire, Gravettien.

KEYWORDS. Art, Prehistory, Venus, Woman, Lespugue, Statuette, Ivory, Gravettian.

\section{Introduction}

L'iconographie préhistorique accorde au vivant, animaux et humains, une place importante, même si les signes, ces graphies géométriques typologiquement plus ou moins élaborées, sont omniprésents tout en étant plus discrets, la plupart du temps moins ostensibles. Le spectacle du vivant sur les parois des grottes et des abris et sur les objets du quotidien durant le Paléolithique récent, entre environ 40000 ans et 10000 ans avant le présent, un vivant auréolé du mystère de sa nature, de ses propriétés et de ses significations, est fascinant. Quand, bien plus souvent que les humains et nous y reviendrons, les animaux nous offrent leur apparente qualité descriptive et figurative, quand ils donnent l'impression d'imiter le réel avec force fidélité ce spectacle devient un émerveillement. Les traitements formels des animaux, leur esthétique, leurs attitudes ou les liens supposément éthologiques qui les lient entre eux semblent parfois si accomplis qu'ils nous conduisent à qualifier l'art animalier paléolithique de « réaliste », voire de «naturaliste ». Et c'est bien la réussite de l'artiste à donner l'air vrai et beau, à représenter avec maîtrise et loyauté le monde visible, qui gouverne encore certaines appréciations des œuvres du passé, comme si elles ne s'inscrivaient que dans une finalité esthétique autonome. Il s'agit là évidemment d'une vieille posture académique héritée d'une conception plutôt radicale de ce que doit être l'art et sa perfection dans les sociétés matériellement développées, c'est-à-dire l'exercice de l'imitation fidèle et scrupuleuse du réel. Force est de reconnaître que les images préhistoriques font voir parfois des 
valeurs plastiques et dénotent des savoir-faire qui les propulsent au rang des œuvres d'art, dans le sens étriqué que l'esthétique occidentale a forgé. Nous comprenons ces images avec nos propres systèmes de codes, et nous compensons leur mutisme en leur prêtant nos goûts et notre esthétique. Et plus ces œuvres sont anciennes, plus notre admiration est décuplée. Mais n'oublions jamais que l'animal représenté est fondamentalement allégorique, même si parfois le traitement de certaines de ses parties nous ferait croire qu'il est zoologique. Alors implacablement, face aux représentations préhistoriques, nous mettons en œuvre une opération de déchiffrement du réel. Impossible de nous en dégager. Nul ne déroge à cette règle naturaliste (dans le sens de l'Histoire naturelle), qui veut que la zoologie et/ou l'anatomie du vivant, son éthologie également, soient à un moment donné convoquées dans la lecture et l'interprétation des représentations animales. Et comment pourrait-il en être autrement, les artistes ayant mis à nu le mécanisme des corps et de la psychologie animale. Fouillant le formidable creuset des images animales, intentionnellement et culturellement sélectionnées, le préhistorien cherche toujours à extraire des bribes d'anatomie ou de comportements disparus. Il tente de faire surgir le vivant à travers le filtre de sa représentation, sans toujours mesurer combien et comment elle traduit la vie. Cet espace ménagé entre la réalité et l'illusion de la réalité et entre l'impression visuelle et l'intention conceptuelle est au cœur du langage de l'art face au monde visible. Si l'artiste soumet l'animal aux lois de sa pensée, il en fait plus encore pour les humains qu'il tire définitivement de leur contexte naturel, comme cela est visible avec la «Dame » de Lespugue, dont la dénomination de «Vénus » affirme l'idée, selon les concepts modernes de l'esthétique hérités de la Renaissance du goût antique gréco-romain, que la représentation du beau est féminine (figure 1).

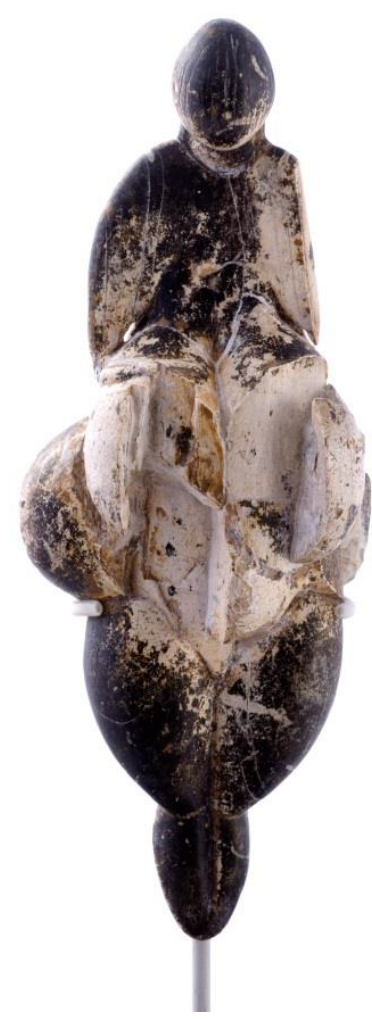

Figure 1. Dame de Lespugue vue de face, (C J.-C. Domenech, MNHN, Musée de l'Homme

\section{Originalité figurative des représentations humaines}

Depuis la première publication de René de Saint-Périer dans la revue l'Anthropologie (1922, p.361-381) beaucoup a été écrit sur la dame de Lespugue. Se succèdent ainsi des savantes et implacables autopsies anatomo-morphologiques ou pathologiques (Duhard, 1993 ; Pales, 1968, 1972), des pages historiographiques précieusement documentées (Hurel, 2013 ; Bertin, 2018), de magistrales descriptions formelles, esthétiques et techno-stylistiques (Coppens, 1989 ; Vialou, 1995 - 
1996), des digressions ethnographiques de type analogiste ou comparatiste et empruntes de concepts racialistes basées sur sa prétendue stéatopygie, qui nous renvoient à l'Afrique du moment chez les Boschimans ou les Hottentots, considérés longtemps comme les images vivantes des peuples préhistoriques, des fossiles vivants en quelque sorte (Luquet, 1934 ; Piette, 1894 ; Saint-Périer, 1922, 1923), des analyses sémantiques toutes faites sur la maternité, la sexualité et la puissance créatrice et génératrice de la Déesse-Mère incarnée par les « vénus » prétendument enceintes, ou sur un mythe des origines ou le mouvement perpétuel de la vie dont l'idole serait l'actrice, des essais où elle est convoquée pour servir la réhabilitation de la place et du rôle de la femme dans les sociétés préhistoriques (Cohen, 2003), et même, enfin dirais-je, quelques envolées lyriques de nature philosophique, poétique ou artistique, aussi libres et subjectives que l'œuvre elle-même (figure 2). Finalement tout ou presque a été écrit sur cette statuette et, il y a peu, un ouvrage lui a même été entièrement consacré (Rouquerol et Moal, 2018), dévoilant selon l'un des auteurs le secret de la « double » et semble-t-il de la même dame de Lespugue et qui ouvre un large champ à la lecture des formes et à leur interprétation, non sans poésie et talent. Pour ne pas répéter à l'infini ou plagier ces innombrables pages, on peut raisonnablement se demander ce que l'on peut encore écrire d'original ou d'inédit sur la dame de Lespugue, l'une des plus célèbres œuvres préhistoriques, dont la dissection formelle et sémantique a été sans cesse repensée depuis 100 ans. Pas grand-chose a priori, peut-être quelques lignes sur la manière dont cette statuette reproduit la nature et tout à la fois la transcende dans une sorte de quête synthétique, comment elle exprime et simplifie la réalité ou reflète la vie et comment en même temps elle s'impose comme une métaphore esthétique d'une rare puissance, pourraient suffire. Nous ne ferons pas l'histoire de sa découverte, nous ne détaillerons pas la nature géo-archéologique du site et de l'habitat qui l'hébergea, ni même les détails de sa plastique moderne et audacieuse. Nous proposerons une brève synthèse de ces item afin de replacer l'œuvre dans son contexte et laisserons le lecteur à sa découverte des articles et des ouvrages qui sont consacrés à cette statuette unique, l'invitant plus simplement encore à venir l'admirer au Musée de l'Homme. Il se fera ainsi une idée personnelle de l'œuvre et s'en délectera à sa manière pour y trouver sa part de réalité, d'idéel et d'esthétique pure (figure 3).

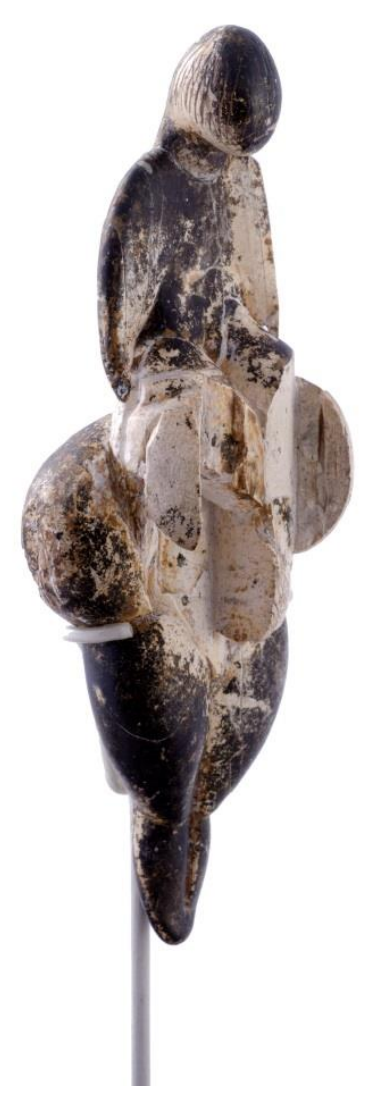

Figure 2. Dame de Lespugue vue de 3/4 avant droit, $\odot$ J.-C. Domenech, MNHN, Musée de l'Homme 


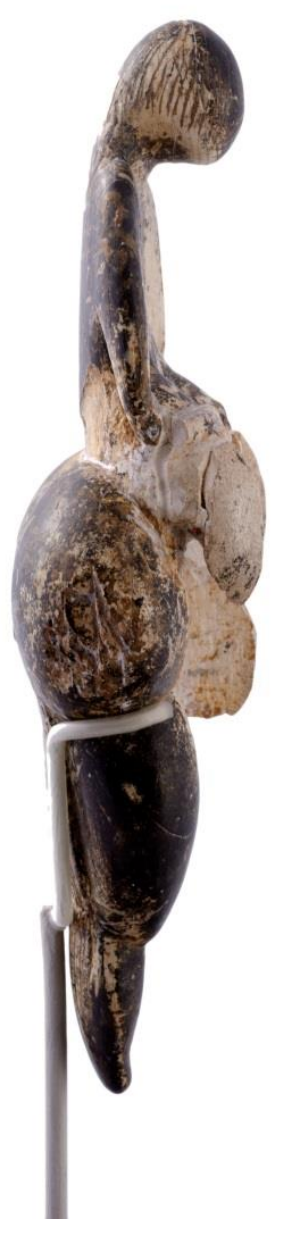

Figure 3. Dame de Lespugue vue de profil droit, (C) J.-C. Domenech, MNHN, Musée de l'Homme

Et c'est bien cette image ambiguë que nous offre la dame de Lespugue et la plupart de ses consoeurs gravettiennes ou plus récentes, l'image de la femme sacrifiée à l'idéal des formes pures, d'une esthétique repensée. Les représentations humaines paléolithiques peintes, gravées ou sculptées, sont souvent extravagantes, déformées ou abrégées. Elles échappent aux normes figuratives qui gouvernent une partie des représentations animales dont le réalisme, souvent exagéré, n'en demeure pas moins affleurant. Aux animaux, les artistes opposent leur image ou celle de leurs semblables résolument anticonformiste et fréquemment segmentaire. Au Paléolithique l'animal acquiert une forme de plénitude expressive. Parallèlement, l'humain, moins fréquent, se situe à part dans un répertoire figuratif qu'il contribue tout à la fois à enrichir et à rendre atypique. Parmi les représentations sexuées, les femmes, complètes ou réduites à la seule représentation de leur sexe, dominent largement le corpus des humains ( $80 \%$ environ), reléguant les hommes à une place marginale pour ne pas dire anecdotique. C'est donc la femme (Delporte, 1979), une femme, qu'il nous est permis de célébrer ici modestement.

\section{La dame de Lespugue, une princesse classée $X$ ?}

Du haut de ses 14,75 cm d'ivoire de mammouth, sculptée dans le vif de la matière essentielle (fraîche ou fossile ?), peu à peu ridée, puis tachée de noir de manganèse au contact prolongé du sédiment, enfin fracturée sous les effets du temps, peut-être de l'usage, mais aussi les aléas d'un coup de pioche malheureux donné à l'arrière d'un «foyer » au fond de la grotte des Rideaux (HauteGaronne), la dame de Lespugue domine depuis probablement plus de 25000 ans l'iconographie féminine gravettienne et particulièrement la statuaire (figure 4). Cette dernière compte à ce jour plus de 120 rondes-bosses sculptées dans l'ivoire, le calcaire, la craie, la stéatite, la calcite, le bois de 
cervidés ou l'os : elles sont parfois modelées dans la terre et cuites. Ces statuettes sont connues dans une trentaine de sites depuis le piémont pyrénéen jusqu'aux rives du lac Baïkal en Sibérie. La grotte des Rideaux, point le plus occidental de l'aire de répartition de ces figurines, avec la grotte du Pape à Brassempouy (Landes), doit son nom aux reliefs stalagmitiques drapant les parois. Elle s'ouvre sous l'ancien château de Lespugue en rive droite et pentue des gorges de la Save, modeste affluent de la Garonne bien connu aujourd'hui pour abriter plusieurs sites paléolithiques remarquables comme les grottes des Harpons, des Bœufs, des Scilles et de Gouërris.

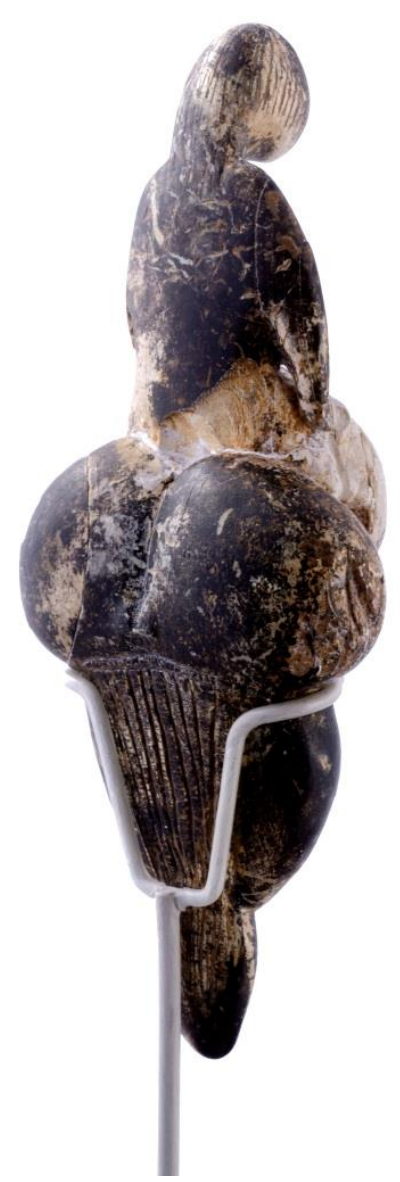

Figure 4. Dame de Lespugue vue de 3/4 arrière droit, (C) J.-C. Domenech, MNHN, Musée de l'Homme

La grotte des Rideaux, creusée dans les calcaires du Coniacien, longue d'une vingtaine de mètres, large de $8 \mathrm{~m}$ et haute de $6 \mathrm{~m}$, est découverte par René de Saint-Périer au mois d'août 1911. Les principales fouilles y sont effectuées jusqu'en 1914. Elles sont reprises en 1922 dans des niveaux situés à l'entrée de la cavité et en grande partie remaniés à l'époque gallo-romaine et lors d'aménagements médiévaux en lien avec le château, puis dans des couches qui semblent en place, protégées par de gros blocs d'effondrements, dans la partie plus profonde de la galerie, entre 6 et 8 $\mathrm{m}$ de l'entrée. Le contexte archéostratigraphique de la grotte des Rideaux est attribué au Gravettien moyen à burins de Noailles, malgré la présence d'artefacts possiblement rapportés à d'autres périodes (Aurignacien et Solutréen notamment). C'est là, dans la pénombre, plantée à $15 \mathrm{~cm}$ de profondeur dans une épaisse couche archéologique noire («foyer »), dans une zone peu riche en vestiges fauniques et lithiques, à environ $6 \mathrm{~m}$ du seuil de la cavité, que la statuette est découverte le 9 août 1922. Un coup de pioche porté sous une roche l'avait sectionné en deux et avait éparpillé une dizaine d'autres fragments d'ivoire, patiemment récupérés au crible par Suzanne et René de SaintPérier. Le nettoyage, la consolidation et la restauration de l'objet sont confiés au chef de l'atelier des moulages du laboratoire de Paléontologie du Muséum national d'Histoire naturelle, un certain M. Barbier, sous l'autorité de Marcellin Boule, après que les Saint-Périer aient fait don à l'institution parisienne de ce trésor de la ronde-bosse paléolithique. Cette dame retrouvait ainsi un corps presque 
complet dont les courbes et les volumes n'ont cessé de faire jaser depuis. Des moulages en furent effectués et un remodelage en proposa une restitution jugée hypothétique et largement diffusée aujourd'hui dans les boutiques de musées (figure 5).

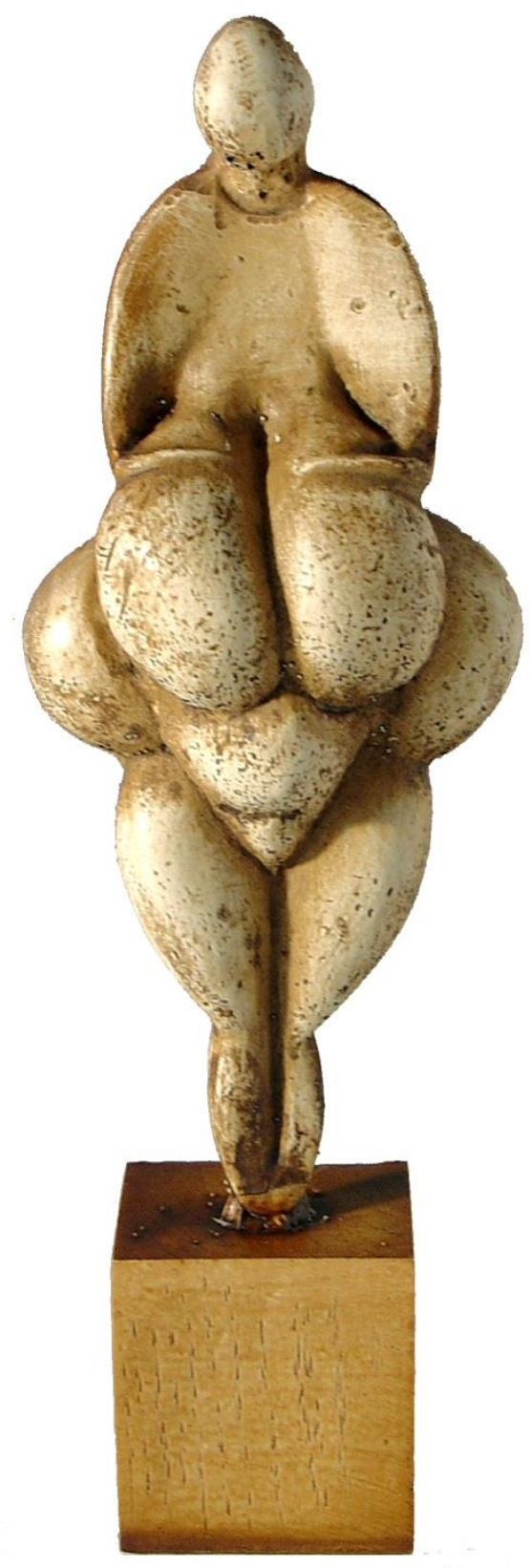

Figure 5. Dame de Lespugue (reconstitution, moulage), () P. Paillet

«Dame», «Vénus », «Déesse » ou encore «Idole », affublée de ces qualificatifs et d'autres encore, cette figuration féminine a pour point cardinal sa nudité presque complète et ses formes spectaculaires.

Elle affiche d'abord d'honnêtes mensurations : $14,75 \mathrm{~cm}$ de haut, $5,8 \mathrm{~cm}$ de large et $3,5 \mathrm{~cm}$ d'épaisseur qui la place parmi les plus grandes rondes-bosses féminines, après la statuette de Savignano (Italie). Mais ce qui frappe d'emblée l'observateur c'est sa forme générale, à la fois adipeuse et efflanquée, massive et longiligne. C'est aussi son aspect lisse, poli, épuré qui impressionne dans ce vocabulaire plastique inédit. Ces canons formels défient les lois de la nature, notamment celles de l'anatomie. Ne cherchons pas le portrait de la femme gravettienne dans cette combinaison puissamment esthétique et hautement stylisée, à la fois abstraite et figurative dans la simplification des formes, d'angles adoucis, de volumes et de proportions conventionnels. Pas plus que nous ne saurions «faire l'anthropologie de la française actuelle en partant des œuvres de 
Picasso ou de Bernard Buffet » comme le soulignait avec une certaine malice André Leroi-Gourhan (1970), nous n'oserions convoquer les statuettes féminines paléolithiques, et tout particulièrement la dame de Lespugue, comme des témoins anthropologiques vrais. D'autres s'y sont consacrés, Edouard Piette en son temps et bien des préhistoriens et des anthropologues à sa suite. Ils y ont vu des répliques féminines fidèles d'une supposée « race » disparue, mais dont on trouverait encore les vestiges vivants en Afrique orientale ou méridionale, fâcheuse conséquence d'un engouement pour un comparatisme ethnographique qui ne cachait pas ses fondements racistes.

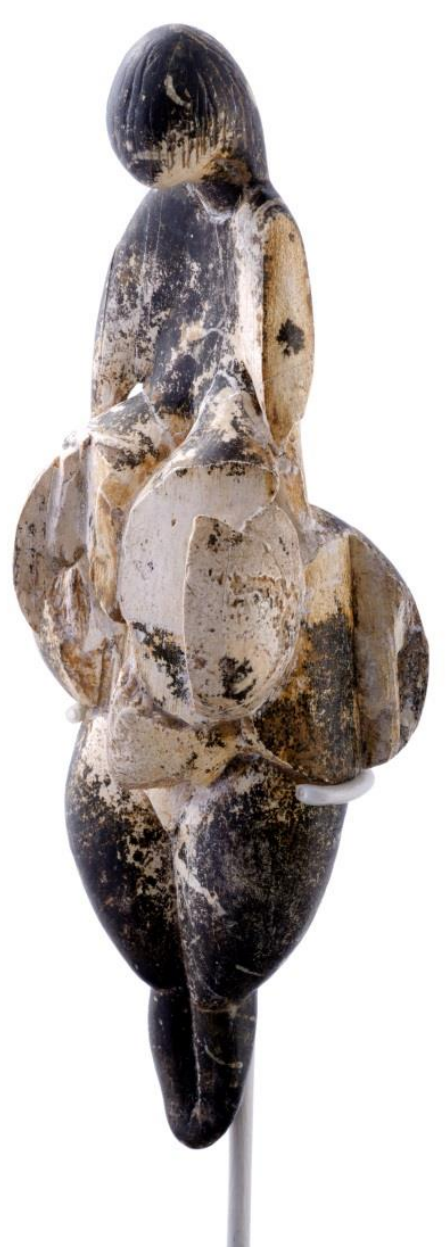

Figure 6. Dame de Lespugue vue de $3 / 4$ avant gauche, (C) J.-C. Domenech, MNHN, Musée de l'Homme

Les seins de la dame de Lespugue, lourdement hypertrophiés et qui s'arrachent du torse fluet, sur lesquels reposent de frêles avant-bras, recouvrent un ventre discret dont ne subsiste aujourd'hui qu'une petite partie (figure 6). Quant au sexe il n'est guère visible, contrairement aux reconstitutions qui donnent à voir un pubis saillant, dépourvu de fente vulvaire, et surmonté, presque effacé par un ventre triangulaire et caréné. La position des bras sur les seins est exceptionnelle dans la statuaire féminine. Notre dame partage cette disposition avec la statuette de Willendorf (Autriche) et peutêtre 2 ou 3 autres représentations de Kostienki et Avdeevo (Russie). Les seins si plantureux trouvent dans le dessin et le volume des hanches, larges et généreuses, qui repoussent les fesses latéralement, le cadre circulaire de leur mise en scène qui sied parfaitement à l'architecture symétrique, mais très insensiblement irrégulière du corps. Un exceptionnel détour par l'anatomie, entorse pourtant inutile pour une œuvre d'art qui en défie pareillement les lois, nous dit que les fesses de la dame de Lespugue, hyper-développées latéralement, sont affligées de stéatomérie ou de stéatocoxie et non de stéatopygie (figure 7), puisqu'elles ne sont pas hyper-développées postérieurement, comme on l'a dit et répété à l'envi en lui trouvant bien des points communs avec Saartjie Baartman et d'autres femmes du groupe ethnique Khoïsan. L'anatomie et la pathologie, si souvent convoquées, n'ont plus 
rien à nous apporter ou nous expliquer sur les glorieuses «monstruosités » artistiques de Lespugue. En revenant à la plastique et à leur nudité de convention les fesses de la dame de Lespugue sont indéniablement callipyges, c'est-à-dire «belles », même si, à l'instar d'Aphrodite soulevant son péplos, elle ne se retourne pas pour les admirer avec superbe par-dessus l'épaule. Bien au contraire, sa petite tête ovoïde sans visage, juste soulignée par une brève chevelure discrètement incisée, est inclinée vers l'avant et le bas dans une posture recueillie (et non satisfaite), comme d'autres représentations russes de Kostienki, Avdeevo, Gagarino ou Khotylevo. Les épaules tombantes et le torse creusé sous le poids des seins et celui d'une insaisissable vie intérieure accentuent ce sentiment de méditation (figure 8). L'humble buste gracieux, élégant et fragile repose sur la prodigieuse sphère mammaire et abdominale et sur l'étourdissant volume pelvien eux-mêmes soutenus par des membres inférieurs très courts et trapus, dont le contour signale avec le buste un semblant de symétrie. Ils sont solidaires de part et d'autre une profonde incision, modelée sur la longueur des cuisses, mais aux lèvres plus fermes et anguleuses, en fuseau, le long des jambes, allusion à peine voilée selon certains à une fente vulvaire absente ou disparue à sa place normale (figure 1). Les cuisses sont épaisses et nettement galbées vers l'avant et latéralement. Sous le pli accentué des genoux, elles opposent leurs convexités massives au dessin des courtes jambes ovales et effilées, prolongées, fusionnées et pincées en languette vers l'avant en guise de pieds à peine suggérés. Contrairement à plusieurs statuettes féminines gravettiennes façonnées dans l'ivoire, les membres sont soudés, mais pour autant ils n'expriment aucune raideur statique.

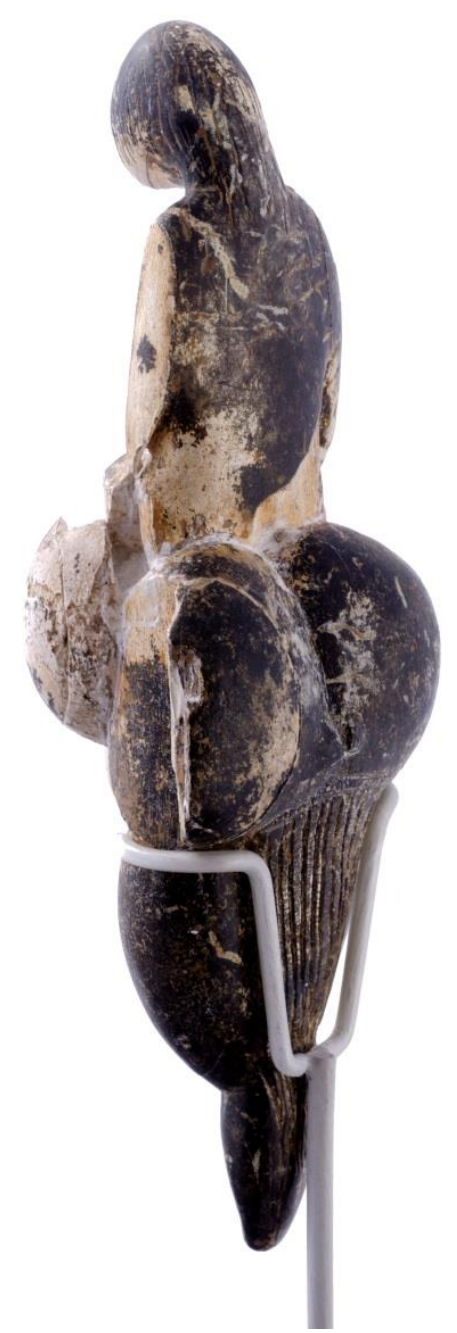

Figure 7. Dame de Lespugue vue de 3/4 arrière gauche, (c) J.-C. Domenech, MNHN, Musée de l'Homme

La beauté plastique de la dame de Lespugue se situe là, subtilement au cour du geste virtuose, dans sa pureté de style entre élégance et pesanteur. Elle est contenue dans la composition parfois 
qualifiée de « cubiste », voire quasiment abstraite dans certains de ses détails à peine esquissés, dans ses amples volumes corporels, lustrés par les usages et sur lesquels notre regard glisse pour ne jamais s'arrêter ou presque. Si ce n'étaient les vieilles fractures du support non réduites, les discrètes incisions de la chevelure et les stries verticales d'un improbable vêtement (pagne ?) qui voile les cuisses et les jambes, agrafé à l'arrière et sous les fesses, la ronde-bosse serait délicieusement lisse, sans aspérité.

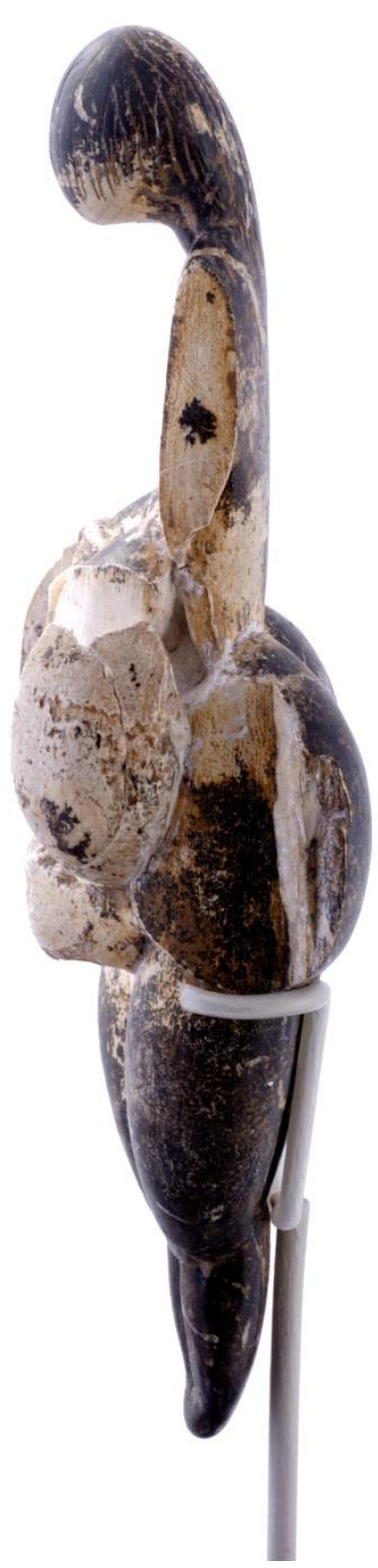

Figure 8. Dame de Lespugue vue de profil gauche, @ J.-C. Domenech, MNHN, Musée de l'Homme

Dans le creux de la main, dont elle possède plus ou moins les dimensions, elle pourrait aussi, nous dit-on, révéler une autre de ses qualités : sa double personnalité. Retournez-là deux fois avec délicatesse, une première pour profiter de sa chute de reins et une deuxième pour la renverser tout simplement (figure 9). Notre vénus ainsi abandonnée devient tout à coup un autre personnage. La chevelure à la place des plis du pagne, la tête dans les pieds et les jambes, le cou dans l'inflexion des genoux et enfin les épaules et le buste dans les fortes cuisses affirmeraient sa bipolarité (Coppens, 1989 ; Luquet, 1934). 


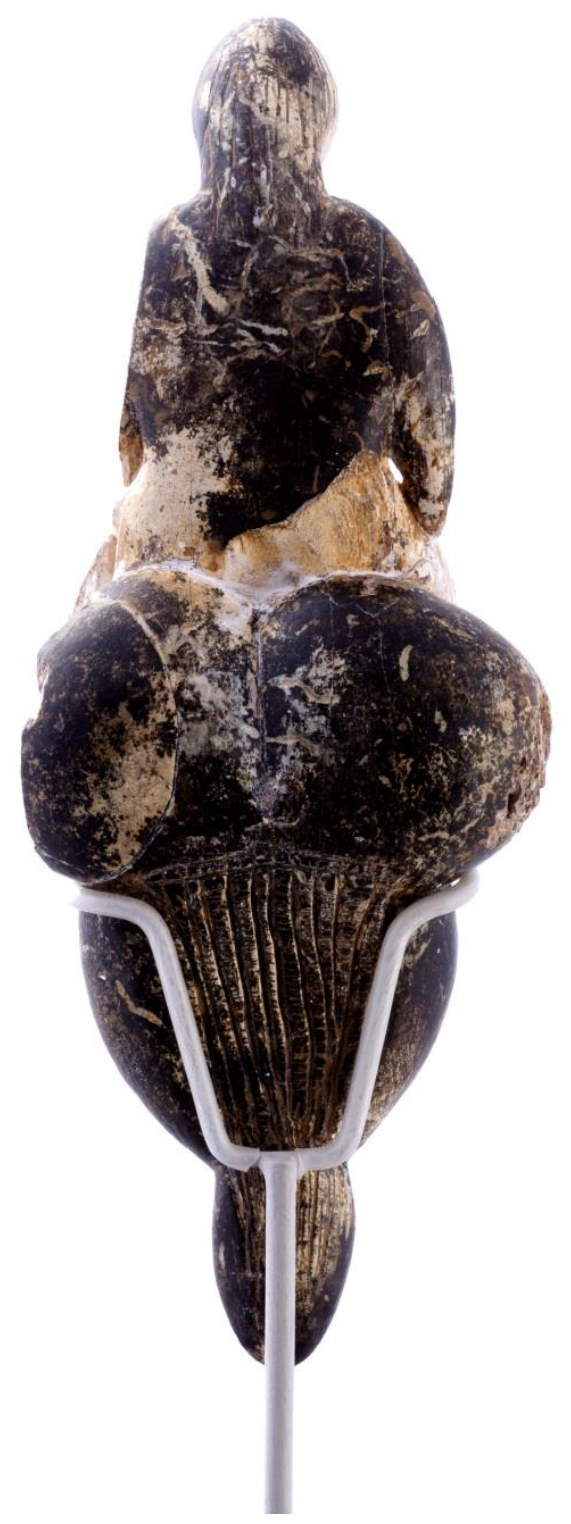

Figure 9. Dame de Lespugue vue de dos, (C) J.-C. Domenech, MNHN, Musée de l'Homme

Les fesses seraient enfin sillonnées dans le bon sens, donnant un peu de vie réelle à cet autre double, gracile et pesant à la fois. La vénus de Lespugue serait aussi double vue de profil, androgyne en quelque sorte. D'aucuns y auraient aperçu une forme phallique, incorporant dans l'œuvre un double genre symbolique en un judicieux effet de paréidolie. Mais il convient de rester prudent en termes d'interprétation de la plastique du nu et de ses multiples lectures.

\section{La dame de Lespugue. Une forme en devenir.}

La dame de Lespugue pose évidemment la question très banale et récurrente de la ressemblance dans l'art. L'apparence réaliste de la combinaison des formes affichées par cette statuette a trompé son monde. Les anthropologues, les préhistoriens, les médecins et tant d'autres se sont essayé à des analyses littérales du corps de la dame de Lespugue et de ses détails, comme s'ils avaient à faire à un être vivant avec ses os, ses muscles, ses graisses si importantes dans ce cas clinique, sa peau et ses phanères. Nous avons également sacrifié un peu dans ces lignes à cette pratique d'usage courant. Mais c'est oublier que le sujet de ces digressions anatomo-physiologiques, souvent improvisées, est une ronde-bosse d'une quinzaine de centimètres nait d'une matière, l'ivoire de mammouth, grâce à l'esprit et à la main d'un artiste sculpteur ivoirier qui a dialogué avec la matière caressée pour en extraire l'âme et lui «donner une existence réelle»(Lemny, 2012). L'artiste a pris possession de 
l'ivoire, et la taille directe a libéré une part de la puissance, de l'énergie, de la vie et du pouvoir mystérieux de cette matière noble et essentielle. L'ivoire a infusé du sacré dans l'objet, en tous cas un esprit à la source de ses formes minimales.

Les représentations sont empruntées à l'expérience et à l'imagination : elles sont cognitives. Ce n'est pas le monde extérieur que les artistes se sont acharnés à traduire. Au reste existe-t-il vraiment, hors de leur regard et plus globalement de leur monde sensoriel ? Dans les faits, ils ont retravaillé, remodelé et fragmenté la réalité en fonction de leur propre vision du monde, de leur monde intérieur ou de celui de la communauté à laquelle ils appartenaient, des messages qu'ils souhaitaient transmettre ou des effets optiques qu'ils souhaitaient produire, de leur propre maîtrise technique et peut-être même de leur inventivité. Et manifestement ces deux dernières qualités ne manquaient pas au sculpteur de Lespugue, dont la personnalité et l'individualité ressurgissent dans le traitement unique d'une forme en devenir qui réalise l'unité de l'esprit et du corps et qui atteint l'essence et le sens réel des choses (Lemny, 2012). Il a donné à son œuvre l'apparence de la vie ou d'une séquence de vie au moment où elle pénétrait le corps tangible du tronçon d'ivoire choisi, au moment où elle s'y fondait. Elle a échappé ensuite à son monopole. Et cette vie nouvelle a progressivement éloigné l'œuvre de la réalité triviale et a facilité ce qu'Ernst Gombrich (1996) définissait comme les mécanismes de la projection, c'est-à-dire la faculté de discriminer les informations à partir d'images indécises ou moins précises. En atténuant les capacités perceptives, ces mécanismes ont donné la priorité au savoir conceptuel sur les processus de la vision et ont même suscité des inventions ou des entorses à la réalité. Ce sont en quelque sorte ces anomalies, ces anormalités dans les images, même les plus subtiles, qui leur permettaient de mieux fonctionner et d'être peut-être plus faciles à mémoriser (Paillet, 2019).

Ces réflexions sur la manière dont le vivant est perçu et choisi, c'est-à-dire observé et en même temps imaginé, et sur la façon dont il est traduit en images, font deviner un peu du sens et de la valeur propres des représentations. La dame de Lespugue nous force à penser et à comprendre, car l'artiste appréhende la réalité à travers une multiplicité de filtres et, en premier lieu, son propre système physiologique sensoriel qui informe son cerveau, libre ou contraint de reconstruire et d'interpréter le monde ainsi proposé. "Quel enfantillage d'ailleurs, de croire à la réalité puisque nous portons chacun la nôtre dans notre pensée et dans nos organes. Nos yeux, nos oreilles, notre odorat, notre goût différents créent autant de vérité qu'il y a d'hommes sur la terre. Et nos esprits qui reçoivent les instructions de ces organes, diversement impressionnés, comprennent, analysent et jugent comme si chacun de nous appartenait à une autre race » (Maupassant, 1888, p.XVIII).

\section{La femme « rabotée " (Devigne, 1920 - à propos de la Princesse $X$ de Constantin Brancusi)}

Depuis sa lointaine origine, la dame de Lespugue continue d'agir hors de ses qualités et de ses intentions premières qui ne nous sont pas destinées. Débarrassée de tout détail superflu, cette œuvre, qui montre, sous la multiplicité des plans et de ses points de vue, une épuration, une simplification et une géométrisation des formes, réalise la première synthèse esthétique pure du corps de la femme, 25000 ans environ avant Brancusi. Sous les coups de silex et sous l'œil de l'artiste visionnaire de Lespugue, qui invente un nouveau langage artistique, la femme lourde par fausse apparence se métamorphose en dame sublimée, élégante et gracieuse, par le truchement d'une reconstruction «insolite de symétries et de volumes entrecroisés» (Vialou, 1995-1996, p.44) et de formes «sphéroïdes, ovoïdes, cylindriques, qui se répondent sans se répéter. Et on découvre, dans un examen plus attentif, que ce sont une tête, un ventre, des seins, des bras, des cuisses. La vénus de Lespugue, c'est une image, mais ce sont d'abord des rythmes [...] Mais le rythme, les rythmes de l'image vous saisissent. C'est comme une fulguration soudaine : un coup de poing au creux du ventre, qui peut provoquer une sorte d'élan sensuel, mystique » (Senghor, 1967, p.11). 
Aujourd'hui au musée, loin de sa demeure d'où elle fut arrachée sans ménagement il y a 100 ans, la dame de Lespugue, idole symboliquement désincarnée, n'affiche plus que son architecture géométrique intemporelle avec une forme d'humilité et une puissance spirituelle, l'étincelle de son esprit, qui nous interrogent et nous fascinent en même temps.

Il est temps à présent de s'abandonner au plaisir simple d'admirer ce trésor moderne de la statuaire préhistorique.

\section{Bibliographie}

Bertin M., 2018. De la sortie de terre à la mise sous verre : la Vénus de Lespugue comme chef-d'œuvre de l'art mobilier paléolithique. Essai de biographie muséale, ORGANON, n ${ }^{\circ} 50$, p.19-43.

Cohen C., 2003. La femme des origines. Images de la femme dans la Préhistoire occidentale, éditions Belin-Herscher, Paris, $191 \mathrm{p}$.

Coppens Y., 1989. L'ambiguité des doubles vénus du Gravettien de France (information), Comptes rendus des séances de l'Académie des Inscriptions et Belles-Lettres, $133^{\mathrm{e}}$ année, N.3, p.566-571.

Delporte H., 1979. L'image de la femme dans l'art préhistorique, éditions Picard, Paris.

Devigne R., 2020. Aux Indépendants. L’homme qui rabote les femmes, L'Ére nouvelle, 29 janvier 1920, p.6.

Duhard J.-P., 1993. Réalisme de l'image féminine paléolithique, Collection Cahiers du Quaternaire, ${ }^{\circ} 19$, éditions du CNRS, Paris, $244 \mathrm{p}$.

Gombrich E., 1996. L'art et l'illusion. Psychologie de la représentation picturale, éditions Gallimard, 404 p.

Hurel A., 2013. Des Bushmen en Europe ? Vénus paléolithiques et «négroïdes » de Grimaldi dans la construction de la Préhistoire française (in) Blanckaert C. (dir.) La Vénus hottente. Entre Barnum et Muséum, Publications scientifiques du Muséum, Paris, p.291-363

Lemny D., 2012. Brancusi. Au-delà de toutes les frontières, éditions Fage, Lyon, 167 p.

Leroi-Gourhan A., 1970. Observations technologiques sur le rythme statuaire (in) Pouillon J. et Maranda P. (dir.) Echanges et Communications, Mélanges offerts à Claude Lévi-Strauss à l'occasion de son $60^{\text {ème }}$ anniversaire, éditions De Gruyter Mouton, La Haye, p.658-676.

Luquet G.-H., 1934. Les vénus paléolithiques, Journal de Psychologie normale et pathologique, n³1, 5-6, p.429-460.

Maupassant G. (de), 1888. Pierre et Jean, éditions Paul Ollendorff, Paris, p.XVIII.

Paillet P., 2019. Itinéraires symboliques à la fin du Tardiglaciaire : continuités et discontinuités figuratives en Europe occidentale, HDR « Sciences de l'Univers, de la Terre et de l'Espace », spécialité « Préhistoire », Muséum national d'Histoire naturelle, Paris, 304 p.

Pales L., 1968. Statuaire préhistorique et anatomie, Bulletins et Mémoires de la Société d'anthropologie de Paris, XIIe série, T.3, fasc.1, p.63-76.

Pales L., 1972. Les Ci-devant Vénus stéatopyges aurignaciennes, Actas del Simposium de Arte rupestre, Santander, p.217-261.

Piette E., 1894. Races humaines de la période glyptique, Bulletins de la Société d'anthropologie de Paris, N5, IVe série, p.381-394.

Rouquerol N. et Moal F., 2018. La vénus de Lespugue révélée. Regards croisés, éditions Locus Solus, 221 p.

Saint-Périer R. (de), 1922. Statuette de femme stéatopyge découverte à Lespugue (Haute-Gronne), L'Anthropologie, T.XXXII, $\mathrm{n}^{\circ} 1-2$, p.361-381.

Saint-Périer R. (de), 1923. La statuette féminine de Lespugue, Revue de Comminges, T.XXXVII, N¹2, p.169-177.

Senghor L.-S., 1967. Qu’est-ce que la négritude, Études françaises, Vol.3, N¹, p.3-20.

Vialou D., 1995-1996. Lespugue, femme ou vénus ? (in) Vialou D. (dir.) L'art Préhistorique, Dossiers d'Archéologie, n²09, éditions Faton, p.32-65. 